\title{
How Journals Treat Scientific Misconduct Allegations: Should Articles Be Retracted?
}

Evidence-based medicine is the basis for most of our clinical decisions. Innumerable researchers worldwide provide us with new data every day. Scientific results are presented at meetings, international conferences, and are published in peer-reviewed journals.

Most of our readers know this process from personal experience as an author, reviewer, editor, or associate editor.

In 2015, an article entitled "Autologous Peripheral Blood Mononuclear Cells as Treatment in Refractory Acute Respiratory Distress Syndrome" was submitted to this journal by a group of researchers. After an independent peer review process by different international reviewers and several revisions, the paper was accepted and published in the 6th issue of Respiration in 2015 [1].

At the end of January of this year, the journal received an email from the Karolinska Institutet in Sweden with the request to withdraw the article. Since 2016, the Karolinska Institute has examined several research projects from the aforementioned group and came to the decision that the research underpinning the article had been conducted without meeting the required ethical standards (Fig. 1). This decision is contrary to the assessment of the Central Ethical Review Board of Sweden, where an expert group analyzed the research and concluded that it does not constitute grounds to determine scientific misconduct.

So, now it is up to the journal to decide on how to respond. First of all, the formal reviewing process followed all the standard international best practice guidelines. As usual, the responsible editor and the reviewers analyzed the available data and subsequently accepted the article. Should the journal and the publisher now withdraw the article? Shall we ignore the request? Or shall we give the authors the option to respond to the request?

After several internal discussions we decided to offer both parties the option of publishing a letter on the issue. We believe that, based on the evidence available to the journal, we are not in the position to make a judgement on the whether or not to retract this article.

Thus, we leave it up to our readers to decide how to treat the article in question and whether to accept its conclusions as valid. On the following pages, you can read the Karolinska Institutet's position as well as responses from Philipp Jungebluth, Paolo Macchiarini, and Bernhard Holzgraefe.

Felix J.F. Herth, June 29, 2018 
Fig. 1. Karolinska Institutet's report of suspected scientific misconduct.

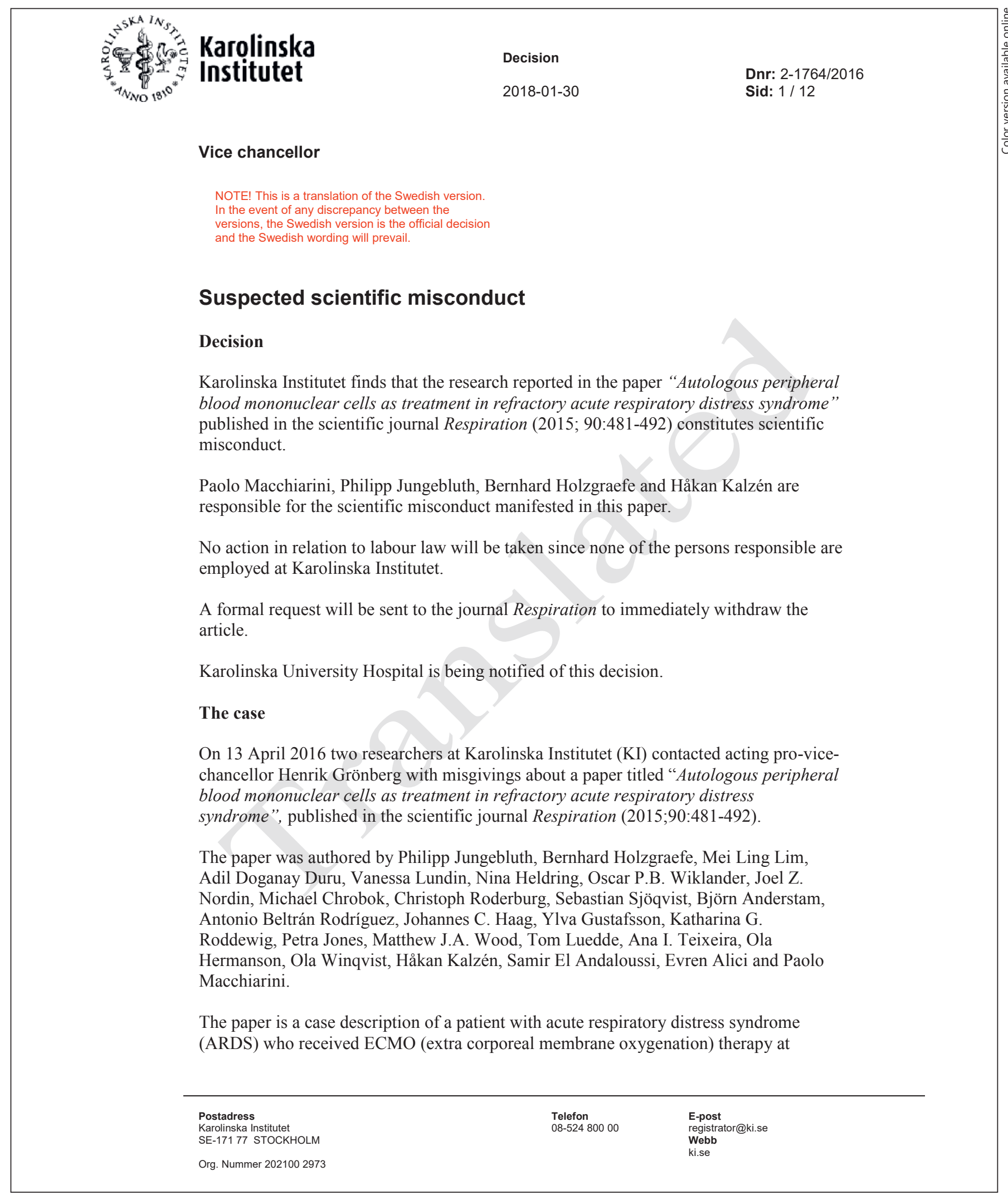

(Figure continued on next pages.) 

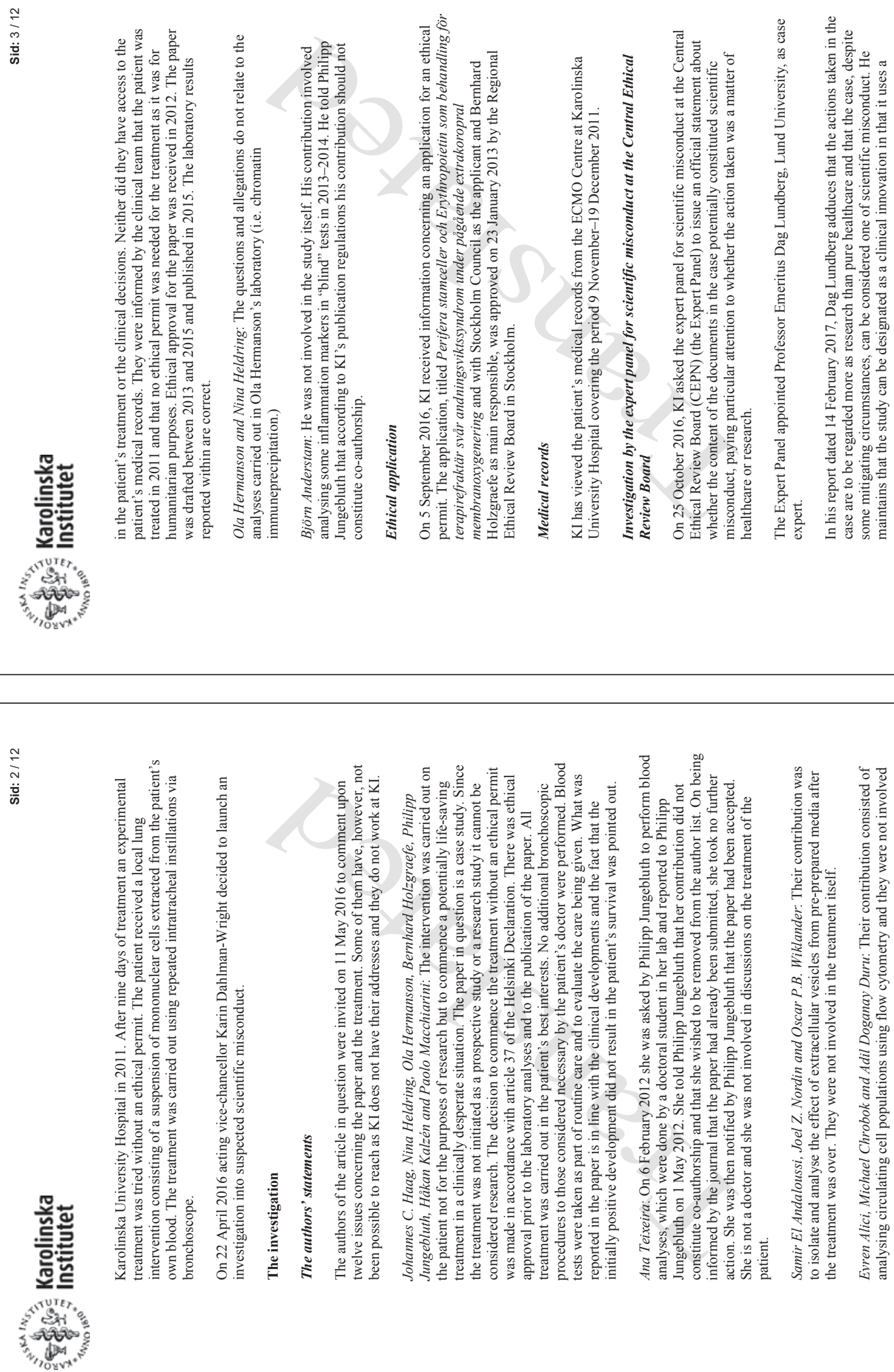

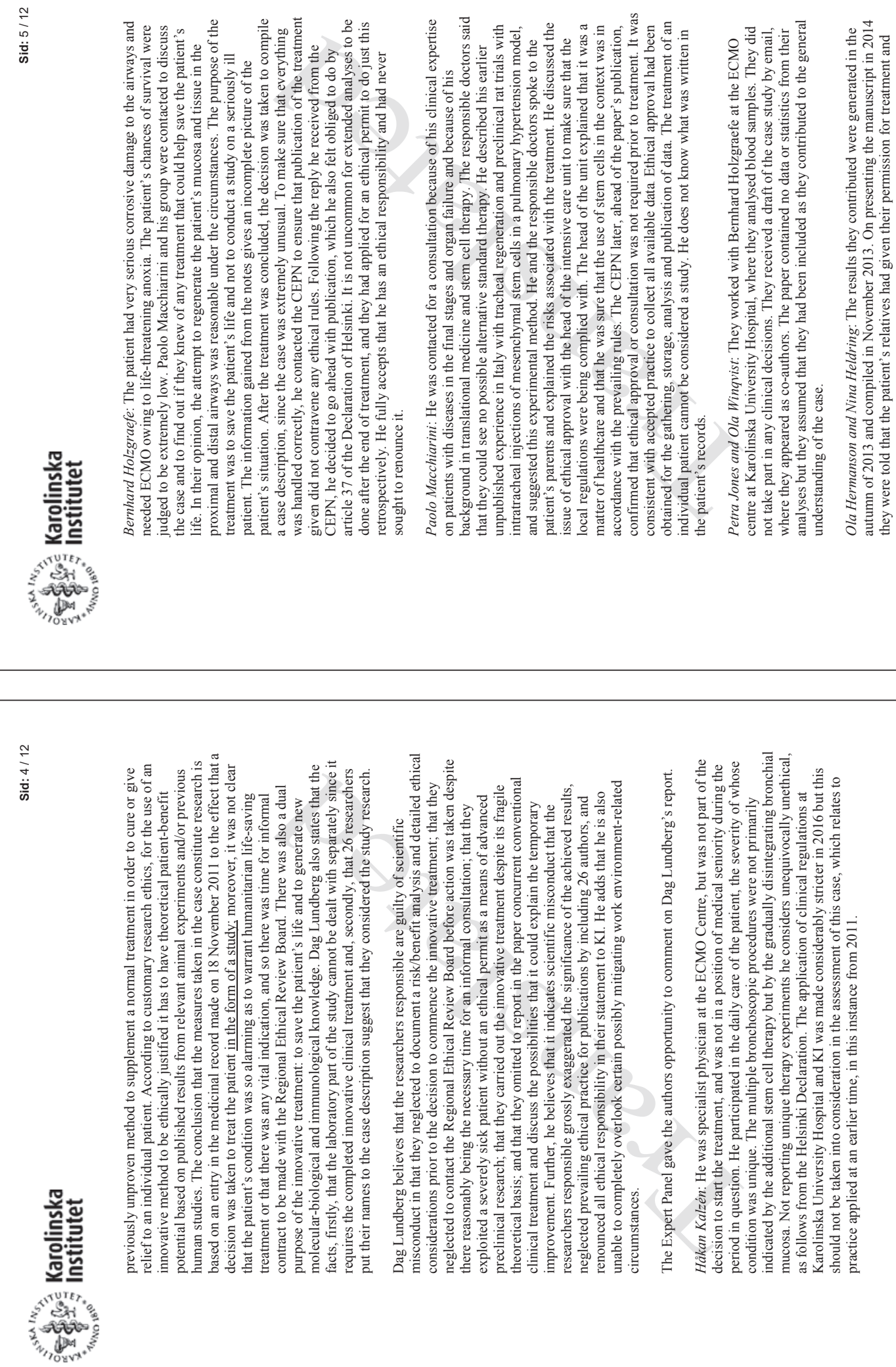

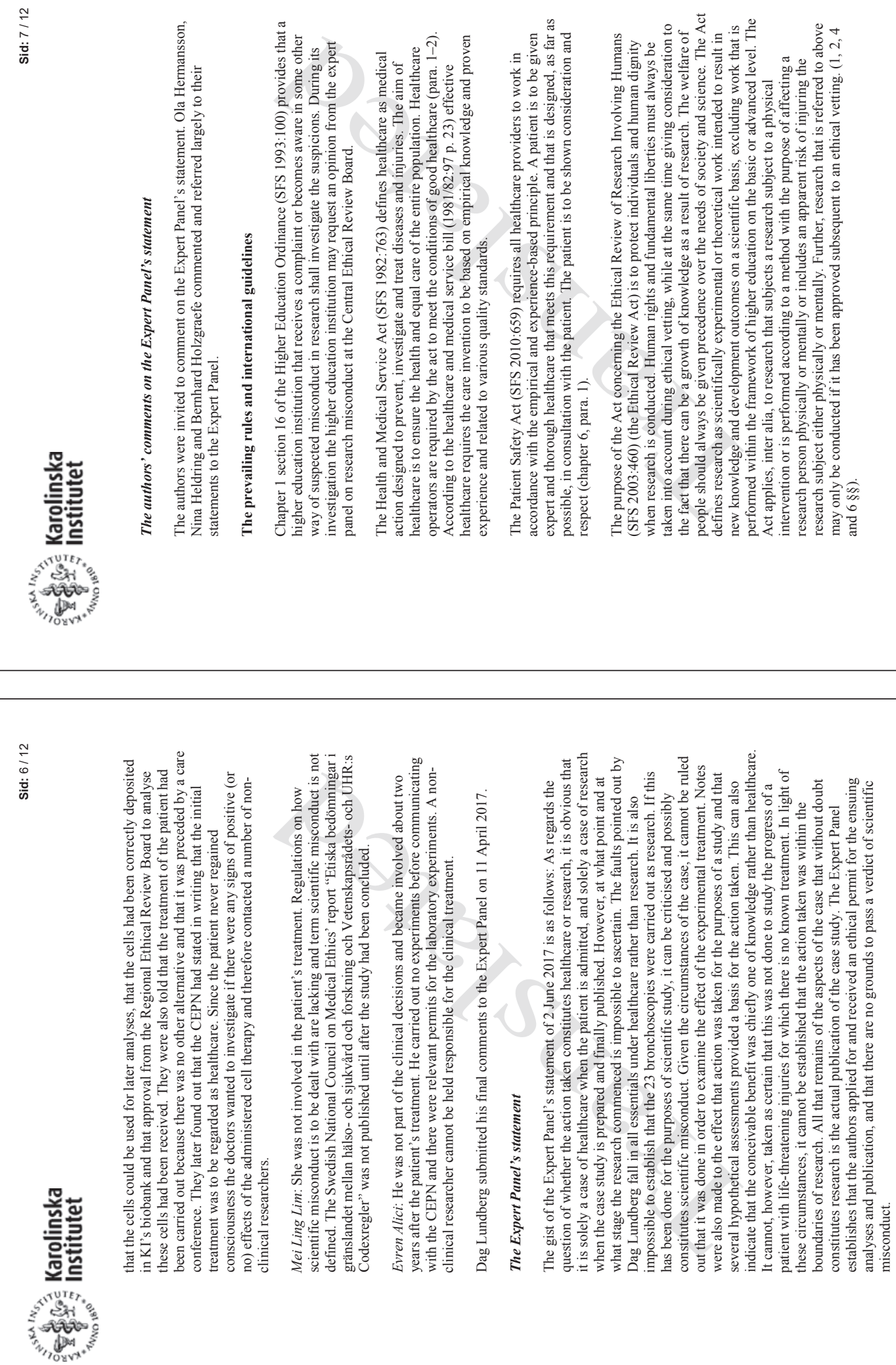

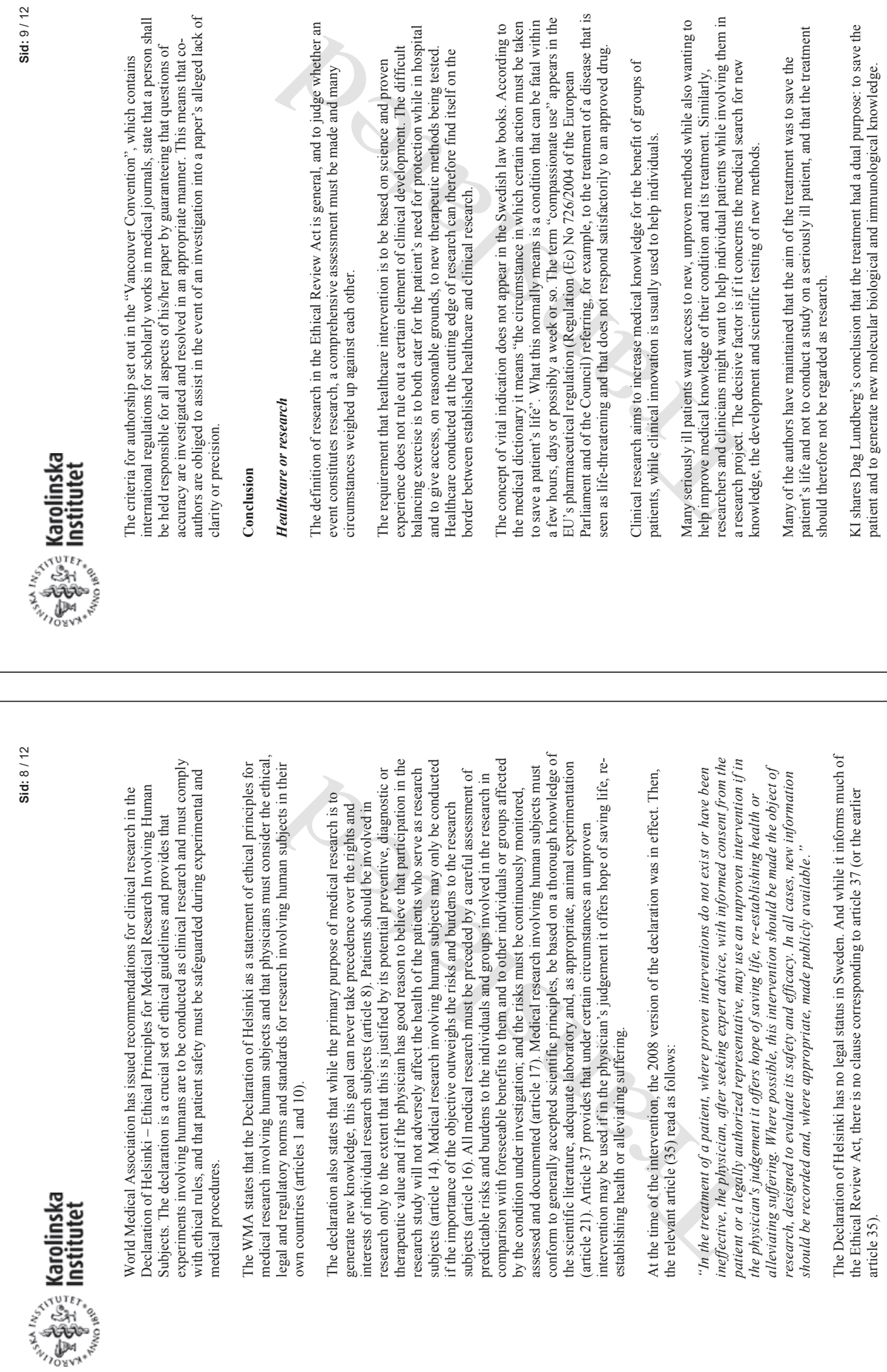

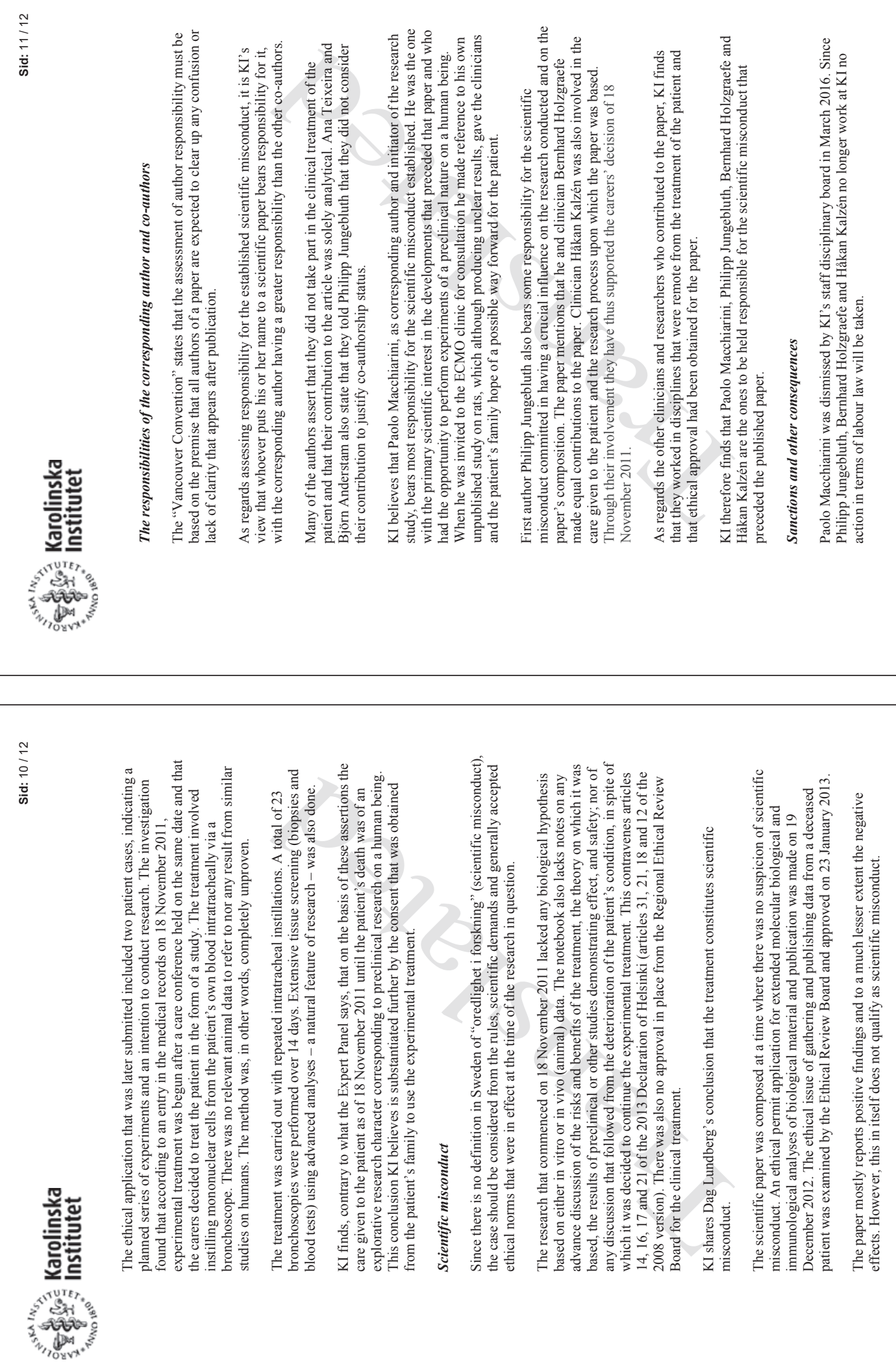


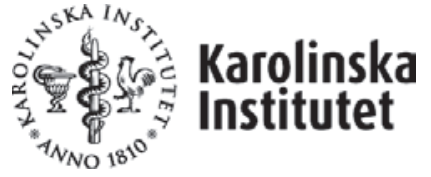

The published paper "Autologous peripheral blood mononuclear cells as treatment in refractory acute respiratory distress syndrome” is based on data produced through scientific misconduct during the basic clinical research process. Even though the published paper as such obtained an ethical permit, the underlying research did not. The paper presents its data in an un-nuanced and overwhelmingly positive manner, which might still entail a risk that other patients will be subjected unnecessarily to similar treatment before adequate preclinical data is available to warrant human studies. The journal Respiration will therefore be urged to withdraw the article forthwith.

The decision on this matter has been taken by Vice-Chancellor Ole Petter Ottersen after presentation by legal counsel Helena Scarabin. Also participating in the final handling of the case was Professor Anders Ekbom and head of the Legal Office Helén Törnqvist.

Ole Petter Ottersen

Helena Scarabin 


\section{Response from Philipp Jungebluth}

Dear Editor,

First of all, I would like to apologize for the trouble our manuscript caused you and your journal. Besides, I want to express my gratitude for the possibility to provide explanation for the ongoing unfortunate matter at the Karolinska Institutet (KI).

I certify that all published data are entirely correct and none of them constitute scientific misconduct. The presented case report (published as "Autologous Peripheral Blood Mononuclear Cells as Treatment in Refractory Acute Respiratory Distress Syndrome”) [1] provides clinical and associated postclinical data of a young patient treated in compassionate use at the Karolinska University Hospital in Stockholm (Sweden) in November 2011.

The following points were considered before the treatment was started and until the publication of the case report:

1 Kenneth Palmer, the former head of the extracorporeal membrane oxygenation (ECMO) center at the Karolinska University Hospital in 2011 contacted us regarding a young patient with a severe inhalation trauma. In a meeting at his unit he asked us whether there would be any treatment on an experimental level that might be helpful for the patient since all conventional therapeutic strategies did not show any beneficial effect.

2 We presented the experimental data that existed at that time (both our preliminary data but also data of other research and clinical groups) and the underlying hypothesis of using autologous progenitor cells and erythropoietin in order to reduce inflammation. Both agents (autologous cells and erythropoietin) are in daily clinical use around the world.

3 The decision to initiate and proceed with the compassionate treatment was then made by the former head of the ECMO center Kenneth Palmer.

4 The approval and informed consent to perform and initiate the treatment was obtained from the parents of the patient after they received a detailed explanation and elucidation regarding the experimental nature of the treatment including all potential risks.

5 The patient showed an immediate improvement to the treatment, and everyone at the ECMO unit was excited by this good response at that time (data included in the paper). Therefore, we decided to share and publish this unique clinical case.

6 We applied for the ethical approval to analyze the cells of the patient and for publication of the data. The ethic committee at KI in Stockholm approved our application.

7 During the submission process, Kenneth Palmer suddenly requested to be removed from the author list of the manuscript for an unknown reason (in light of the subsequent developments, his request seems odd).

The decision that has now been taken by the KI is purely politically motivated. The explanation for their decision is poor and the accusations they made are false and incorrect. KI handles similar cases obviously dissimilar and arbitrary. Other groups at KI ("In vivo Effects of Mesenchymal Stromal Cells in Two Patients with Severe Acute Respiratory Distress Syndrome" [2]) perform similar systemic cell-based treatments (partly without any scientific background), but our local application of autologous progenitor cells and erythropoietin comply with scientific misconduct.

The whole conflict at KI started when Dr. Grinnemo, a former cooperative partner and group leader at KI stole and misused data from our group in 2013. In the following, he was proven to be guilty of scientific misconduct for using our data without approv- al by the KI Ethics Committee. He took revenge by accusing us of unethical scientific and clinical behavior.

Since then, various accusations have been made against our group, and clearly Karolinska Institutet applies different levels of justice in similar cases.

Everything around the treatment and the preparation of the manuscript was correctly done by us. We followed all existing guidelines at Karolinska Hospital and Institutet at that time, but suddenly after alleged accusations against us and a reevaluation of our work, the manuscript constitutes scientific misconduct.

Karolinska University Hospital and Institutet put a lot of pressure on various co-authors of the manuscript who are still working at KI in order to keep them silent and not to speak up. It is a scandal how they protect the University's credibility regardless of the costs for individual scientists.

Whether one should have performed such an experimental treatment in a patient that did not respond to conventional treatment may be disputable and must be further discussed in order to move forward in science and medicine. The decision for the treatment in November 2011 was made by the responsible medical doctors of the department and based on the existing scientific knowledge at that time. I am still convinced by our therapeutic hypothesis and do not see any relevant risk for a patient by the local application of his own cells and erythropoietin.

No matter what one may think about the ethical aspect of such a treatment, there cannot be found any scientific misconduct in our publication and the respective data.

In summary,

1 The clinical team did all in their power to maximize the chance for this patient who was in a severe state and did not respond to conventional therapy.

2 After seeing the clinical improvement, the postclinical team tried to understand parts of the mode of action of the therapy to share it with the scientific community.

Dr. Philipp Jungebluth, February 28, 2018

\section{Response from Paolo Macchiarini}

Dear Editor,

Thank you very much for the opportunity to state my point of view regarding Karolinska Institutet's (KI) request, after requesting opinion from the Swedish Central Ethical Review Board (CEPN) to retract the manuscript "Autologous Peripheral Blood Mononuclear Cells as Treatment in Refractory Acute Respiratory Distress Syndrome" published in Respiration [1].

I cannot agree more with the Editor's and publishing house's request (and wish) to provide a platform for discussions and a forum for a scientific debate. Furthermore, I agree that the background of the conflict between the two parties should be transparent to the readers and audience to enable them to form their own opinion.

Dr. Jungebluth wrote about the dynamics that led to KI's investigation. I shall here start with a clarification of my, and my team's, involvement with this case and my opinions thereon.

\section{Initial Contact}

I was asked for a clinical consultation because of my clinical expertise in patients with end-stage diseases and organ failure - I 
was not employed by the Intensive Care Unit (ICU) but by the Ear, Nose and Throat (ENT) Department - and because of my background in translational medicine and stem cell therapy. The clinicians in charge of the case (e.g., the head of the extracorporeal membrane oxygenation (ECMO) department Dr. Kenneth Palmer) confirmed that they had done their very best and could not see any possible alternative standard treatment, leaving only the option of discontinuing the ECMO - the patient was already on venous-arterial ECMO and had been for several days, a therapeutically applicable recourse only in patients where all standard treatments fail. Not only was there no improvement, but also a worsening of his health status was documented, especially in both lungs (ischemic lungs and upper airway). I talked about our previous, unpublished clinical experience in Italy with the regeneration of the airway and the preclinical rat experience with intratracheal injection of mesenchymal stromal cells in a pulmonary hypertension model [3], and they were interested. The head of the ICU (Dr. Kenneth Palmer) asked me to present this technology and preliminary results, which I did, immediately, to the staff members of the ECMO unit. These were dedicated doctors, trying to find a way to save a dying patient's life.

The clinicians talked first to the parents and then asked me to talk to them. I did, and because of the Swedish language, other members of the team translated whenever it was necessary. Since this was such a dramatic and difficult case, I happen to remember very well that I told the parents that I couldn't possibly know what the outcome would be and that the alternative - to continue the ECMO until the almost certain conclusion of the patient's death had been explained before to them by the clinicians in charge. I explained about the risks of the procedure (intratracheal injection route, impossibility of taking bone marrow because the patient was fully heparinized, risks of airway bleeding, insufficient number of cells taken from the peripheral blood, etc.). I was given to understand also that extra care should be taken with the collection of data since the injuries were sustained whilst the patient was at work and therefore may be needed in subsequent legal action.

I had to leave the country after this initial consultation, but had no concerns about the subsequent care of the patient or collection of the clinical information - I was, indeed, kept updated via telephone and email exchanges.

Obviously I have no knowledge of what was written in the medical records in this case (and I was not asked, as is usually demanded in other countries, to write about my consultation in the medical records, but I speak and write no Swedish, so perhaps that was the reason; besides, I had no access permission to the medical records for patients in Sweden - this has been verified by an internal investigation and is publicly available upon request at the Karolinska University Hospital), but it cannot possibly be taken to mean that I, or my team, considered our suggestions for ways to save the life of this patient as part of an emergency consultation in any way "a study." I shall discuss this in more detail later.

\section{CEPN Critiques about Clinical Indications}

One of the major critiques of the CEPN was that it was not evident that the patient's condition was of such an alarming nature that it acutely motivated compassionate treatment, or that there was a vital indication for treatment, and there was thus time to have informal contact with the regional ethical review board.

Evidently, a patient on ECMO is in a life-threatening condition, especially if ECMO does not yield the expected effects, and there are very strict guidelines for its implementation (Extracorporeal Life Support guidelines). I was shocked to read that Prof. Lundberg (external expert of the CEPN) felt that he can take the view that "...it was not evident that the patient's condition was of such an alarming nature" having merely read the medical records, against worldwide accepted ECMO indications and the opinion of the patient's healthcare providers who were experienced and dedicated ECMO (and advanced ICU) specialists including the head of ECMO, and running one of the biggest and most experienced ECMO Units in Europe, who were alarmed enough to reach out for a consultation with someone from another department in an attempt to save the patient's life.

As for the need for contact with the regional ethical board, during the consultation with the clinicians on the 18th of November, I actually discussed this matter with the head of the ICU (Kenneth Palmer), in order to satisfy myself that local regulations were respected. He expressed that this was simply a healthcare decision in these circumstances, and he was also sure that the use of cell therapy in this context was within all guidelines. CEPN (Mr. Sviden and other authorities) confirmed that this was the correct mode of practice, when we checked before publication.

\section{Innovation versus Healthcare}

Secondary to the health of the patient, ALL "innovative" treatments should serve such a double purpose. In attempting to save a patient's life, we should always - as long as it is of no detriment to the patient, and consent is given - collect as much data as possible to inform future decisions. However, this does not conflict with the fact that this was an acute case in which every opinion was sought in order to attempt to save a patient's life, all conventional options having been tried.

The opinions that Prof. Lundberg expressed in his report about the presumed priorities of the teams involved in the care of this patient are totally disrespectful of the Hippocratic Oath. All doctors have, as their number one priority, their patient's health and wellbeing. I actually feel that the healthcare providers of this poor boy, who attempted every possible solution to this tragic acute injury, should be congratulated for their efforts. They were desperately searching for a possible last therapeutic resource knowing that the situation was catastrophic.

CEPN also stated that the laboratory part of the study cannot be considered separately as it is conditioned upon the completion of the innovative clinical treatment. I am still unsure what Prof. Lundberg means by "cannot be considered separately" - perhaps he means that any failings he perceives in the clinical work means that all authors, regardless of their role, are equally guilty with regard to those failings? If so, then clearly this is absurd. Ethical approvals for the later laboratory part of the study were obtained separately, as they require separate kinds of permissions (e.g., storage and analysis of blood samples). Many researchers needed to be involved in laboratory analysis who were not clinical, and would not have been in contact with the patient, and vice versa. Of course, therefore, there is separation between the roles and responsibilities of different authors, and different legal and ethical requirements for different aspects of what is covered by the manuscript.

\section{Too Many Co-Authors}

Prof. Lundberg stated that "As many as 26 career researchers have put their names to the case study involved, which would indicate that they considered the co-authorship as a potential merit and that they, at the time, considered the study as research." 
Later analysis of the samples (it was late because we had to have all the legal requirements and permissions in place, and it took some time - understandably - to contact the parents to ensure their informed consent, outside of the traumatic circumstances of the acute healthcare emergency) does not imply that that the initial impetus for the procedure was anything other than an attempt to save a patient's life, if this is what is meant by this point. In fact, applying such logic to any postevent analysis quickly shows how absurd such reasoning would be (does carrying out an autopsy imply that the pathologist considered the death part of a "study"? No, it means that he or she was trying to learn from an event).

In fact, the merits of the report were appreciated by the reviewers of the manuscript, who recognize the need for such data and such experimental attempts to save the lives of patients with this devastating condition.

\section{Scientific Misconduct}

Prof. Lundberg then states, "I find that the following indicates that such misconduct has taken place. The responsible researchers have, presumably due to gross negligence:

1 Failed to document a risk/benefit analysis and the associated ethical considerations prior to making the decision to start the innovative treatment."

I cannot comment on what was, or was not, documented after the consultation on 28th November, although given the condition of the patient and the fact that the clinicians stated that all conventional treatments had failed, the potential benefits would have outweighed the risks to such an extent that it may not have seemed necessary to document them.

This appears to be a criticism of the healthcare records held at Karolinska University Hospital, and not of the manuscript or its authors (the "responsible researchers" as Prof. Lundberg phrases it). I do not, however, agree with such criticism. I here attach my email correspondence with the authorial team, requesting documentation of the ethical approvals that were sought at each stage of both the treatment and the research. You will see in this email and its attachments (the ethical approval from CEPN, the biobanking permissions) that each of these was addressed satisfactorily. CEPN, as has been mentioned before, confirmed that in this case ethical permission was NOT required for the treatment, but only for publication (which was subsequently sought and granted). The correct permissions were also held for all other parts of the process (see above).

2 "Failed to contact the regional ethical review board, despite reasonably having time for an informal consultation."

It is Prof. Lundberg's own retrospective assessment that there was time (and need) for such a consultation, against the decision of the head of ECMO and the clinical staff in charge of the case at the time, as I have already pointed out. As also already stated above, contact retrospectively with CEPN confirms that in this case, consultation/approval with the regional ethical review board was not necessary.

3 "Used a severely ill patient, without ethical approval, as a means to carry out advanced preclinical research."

This is an extremely serious accusation, made without any justification. To allege that any medical professional would "use" a patient is beyond comprehension. This statement is defamatory in the extreme.

4 "Carried out the innovative treatment, despite its insubstantial theoretical foundation and insufficient knowledge from ade- quate animal testing. Extrapolated a hypothesis in several steps regarding a potentially beneficial anti-inflammatory effect from the treatment with no scientific evidence. It was thus assumed that mononuclear cells from the blood have the same effect as stem cells from bone marrow and that local intratracheal installation is equivalent to intravenous administration." As Prof. Lundberg himself accepts, there is evidence that stem cells from bone marrow have an anti-inflammatory effect, but the patient's condition precluded a bone marrow aspiration. Attempting to use mononuclear cells from his blood was the only (see above explanation) option that we could give. Given his life support system, as Prof. Lundberg also acknowledges, intravenous use brought considerable risks, and, for example, our previous preclinical and experimental experience in Italy and Spain of intratracheal use gave us the hypothesis that this would be a safer alternative with a chance of efficacy. Indeed, the manuscript itself demonstrates that the hypothesis has some support and an antiinflammatory therapeutic effect was reported - as is well acknowledged by the expert reviewers.

5 "Failed in the article to account for parallel conventional clinical treatment and discuss the possibility that such treatment could provide an explanation for the temporary improvement in the patient's status."

Had any peer reviewer requested any further details about the patient's treatment then we would happily have supplied them, and should the journal wish, we could add such information. I cannot see how this can be listed as grounds for "scientific misconduct."

6 "Considering the minor evidentiary value of the unverified study, strongly exaggerated the significance of the achieved results, which could evidently mislead a less critical reader."

Prof. Lundberg may believe the wording of the manuscript is inappropriate, but the reviewers of it were of a different opinion, and appreciated what we were attempting to do for this patient, and our reporting of it in order to pass knowledge on to the scientific community. It is not uncommon for peers to differ in their opinions on published manuscripts, but I would dispute his single opinion of "strong exaggeration," and place it against those of the expert peer reviewers. There was no misrepresentation of the facts of the case, nor, I believe, were our conclusions misguided.

7 "Neglected the established ethical publication practice by including 26 authors: an unreasonable number, considering the contributions of the individual researchers and the insubstantial scientific value of the publication."

This is the beauty of the multidisciplinary effort to save patients (clinically) and provide international scientific collaboration (some co-authors are outside Sweden because they did specific evaluations requiring expertise and equipment not available in Sweden). It has been always my philosophy that all members that have contributed to a work should be acknowledged, and there is nothing "unethical" about that. The detail of the contribution of each author could be listed should this be deemed necessary. In my personal opinion, it is far more unethical to exclude involved researchers from the author list, in order to increase your own presumed contribution.

9 "Denied all ethical responsibility in their statements, relaying the blame by systematically and categorically referring to other clinically active colleagues. This does not strengthen their case." 
As I have already stated, perhaps Prof. Lundberg was not aware of the responsibilities and situation in this case. I hope that my explanation above clarifies it.

Questions about the clinical record-keeping or the clinical decision-making in this case must be answered by those responsible for those records or decisions. As I say, I do not believe that any wrong decisions were made by those responsible (as confirmed by CEPN when checked), so there is no "blame" to be "relayed." However, since I did not make those decisions, nor see (let alone write) the medical records, I simply cannot answer detailed questions about them - as stated in the previous response.

As I have also said, I believe that the clinical team in this case deserves congratulations (and gratitude) for their attempt to beat the odds for this patient. Such denigrating criticism of people who attempt to save the lives of dying patients does nothing to serve the needs of patients. If this had been your son, what would you have liked the team to do? And, after they had attempted their very best, how would you like them to be treated by the establishment and their peers?

I also feel that I have to comment on Prof. Lundberg's statement that: "A wave of somewhat unbridled enthusiasm for stem cell treatments, including the type that was so forcefully promoted by Prof. Macchiarini, washed over all the units of Karolinska University Hospital at the time of the study. The fact that Prof. Macchiarini at the time had the express support of the KI management obviously reinforced his local authority. It would only be human in that situation if clinically active researchers were to find it difficult to keep a level head and decline an offer to collaborate with him."

Prof. Lundberg states that he retired in 2006 and hence has no experience of the "somewhat unbridled enthusiasm" he claims was "washing over all the units" of the Hospital in 2011. Clearly, he has been exposed to too many colorfully worded media reports about my work and my apparently irresistible and malign influence. Nevertheless, Prof. Lundberg unconsciously ignores the fact that KI had already by that time an excellent, long-lasting and wellpublished history of clinical stem cell application and research (e.g., Prof. Katarina Le Blanc, Prof. Outi Houvatta, etc.).

The truth in this case is that a patient was dying, and his doctors were keen to try anything to save his life. I suggested an experimental option, and everyone, including the patient's family, were aware what the chances of success were. CEPN themselves retrospectively confirmed that these decisions, taken with the patient's best interests at heart, did not require any ethical consultation or approval before initiation of the attempt. It was good practice to collect all the data possible in this case, and all ethical permissions were granted for the collection, storage, analysis, and publication of these data.

Prof. Lundberg and KI may criticize the wording of the manuscript, but there are no grounds for accusations of scientific misconduct. Indeed, I believe the clinical team deserves praise and thanks for their efforts to save a patient's life, and I believe the laboratory researchers did good, careful analyses in order to try to learn from this case and pass the knowledge gained to others who may find themselves with a similar case to deal with.

I shall end by remembering KI vision (https://ki.se/en/about/ startpage), which all our efforts were made to confirm: "Our vision is to make a significant contribution to the improvement of human health; our mission is to conduct research and education and to interact with the community." It is totally in line with the spirit and content of the present manuscript!

How Journals Treat Scientific Misconduct Allegations
And, last but not least, the different modus vivendi of KI and Hospital is worth a few lines. The decision from KI regarding this particular Respiration manuscript work is a clear demonstration on how individuals are treated differently. A similar case (KI referenced 2-2336 and 2-2259, these are public documents) which was reported to KI in 2015 was not properly investigated and KI decided that there was no misconduct. The authors of that study state to have performed "first-in-man" study using a specific population of mesenchymal stem cells for the treatment of ARDS. Before the treatment they had neither performed any animal studies nor applied for ethical permission for this treatment.

Most likely, the following paragraph from the KI's recent decision against our work applies to these researchers as well: "The patient record also lacks notes on any advance discussion of the risks and benefits of the treatment, the theory on which it was based, the results of preclinical or other studies demonstrating effect, and safety."

However, it appears as if $\mathrm{KI}$ in that case did not care to investigate the patient charts. Why the discrepancy in case handling maybe simply because I am now persona non grata and the official (and foreigner) scapegoat of KI's oceanic deficiencies and autoestimation? Of note, I acknowledged receipt of the CEPN verdict submitted about this paper - two and a half weeks after KI made a press release about the receipt of these questions and claimed that they were alleging "research misconduct." KI appears therefore to have abandoned any attempts at compliance with the internationally accepted requirement for any accusations of misconduct to be investigated in a confidential manner, to ensure fairness to all parties.

Prof. Paolo Macchiarini, March 8, 2018

\section{Response from Bernhard Holzgraefe}

\section{Dear Editor,}

I am grateful for the opportunity to explain my point of view regarding the article "Autologous Peripheral Blood Mononuclear Cells as Treatment in Refractory Acute Respiratory Distress Syndrome," which was published in Respiration [1]. The paper is a case report describing an experimental treatment with endotracheal applied autologous peripheral blood-derived mononuclear cells (PBMCs) in a patient suffering from severe acute respiratory distress syndrome treated with invasive mechanical ventilation (IMV) and venoarterial extracorporeal membrane oxygenation (va ECMO). IMV and ECMO were initiated because of a burn-induced airway damage caused by inhalation of hydrated lime. The patient was initially on venovenous ECMO (vv ECMO), but the circuit had to be changed to va ECMO because of hypoxia-induced circulatory failure. The patient was treated at the ECMO center of the Karolinska University Hospital, Stockholm, Sweden.

In the same year, another paper was published by researchers from Karolinska Institutet (KI) about 2 patient cases treated with systemically administered mesenchymal stromal cells during vv ECMO [2].

As one of the co-authors I have been accused of scientific misconduct in 2016 when it was suggested that the treatment was initiated as a research study without approval of the local ethical committee. An investigation was launched by the KI. The KI requested a statement of the expert group on misconduct in research at the 
Central Ethical Review Board of Sweden (Centrala etikprövningsnämnden - CEPN), which appointed a special advisor in this matter. The advisor judged that, based on his analysis, the responsible researchers are guilty of misconduct in research. However the CEPN "considered that it is not possible to establish that the measures have been implemented within the framework of research." "The deficiencies, which the advisor highlighted, have occurred within the care of the patient" and "that these essentially lie within the framework of healthcare and not research" [4]. In contrast to the expert group statement, the KI decided in January 2018 that 4 authors were responsible for scientific misconduct [5]. KI's decision was in my opinion mainly supported by the fact that there was no approval from the local ethical committee for the treatment with PBMCs (Method section 1).

At the time of treatment (2011), I was employed as a consultant/attending physician at the ECMO department of the Karolinska University Hospital, and I had started my doctoral (PhD) studies at the KI in May 2011. My role during the treatment was that of a medical doctor (and not as a researcher) who was part of a team treating this patient. After the patient had died, it was decided to write a case report, and I applied for ethical approval, biobanking, and a patient data registry for writing this case report. We also received written informed consent from the next of kin for this. Here, I had the role of a researcher and all necessary approvals were obtained as stated by the CEPN [4].

It has never been my intention to perform a clinical experimental study in a severely sick patient without ethical approval, which I have been accused for. I was working as a physician in the ECMO unit and delivered medical care to the patients, which in this particular case included the PBMC treatment. Furthermore, I was not authorized to take a final decision to start the PBMC treatment.

Bernhard Holzgraefe, April 4, 2018

\section{Disclaimer}

The statements, opinions, and data contained in this Editor's Note are solely those of the individual authors and contributors.

\section{References}

1 Jungebluth P, Holzgraefe B, Lim ML, Duru $\mathrm{AD}$, Lundin V, Heldring N, Wiklander OP, Nordin JZ, Chrobok M, Roderburg C, Sjöqvist S, Anderstam B, Beltrán Rodríguez A, Haag JC, Gustafsson Y, Roddewig KG, Jones P, Wood MJ, Luedde T, Teixeira AI, Hermanson O, Winqvist O, Kalzén H, El Andaloussi S, Alici E, Macchiarini P: Autologous peripheral blood mononuclear cells as treatment in refractory acute respiratory distress syndrome. Respiration 2015;90:481-492.

2 Simonson OE, Mougiakakos D, Heldring N, Bassi G, Johansson HJ, Dalén M, Jitschin R,
Rodin S, Corbascio M, El Andaloussi S, Wiklander OP, Nordin JZ, Skog J, Romain C, Koestler T, Hellgren-Johansson L, Schiller $\mathrm{P}$, Joachimsson PO, Hägglund $\mathrm{H}$, Mattsson M, Lehtiö J, Faridani OR, Sandberg R, Korsgren O, Krampera M, Weiss DJ, Grinnemo KH, Le Blanc K: In vivo effects of mesenchymal stromal cells in two patients with severe acute respiratory distress syndrome. Stem Cells Transl Med 2015;4:11991213.

3 Jungebluth P, Luedde M, Ferrer E, Luedde T, Vucur M, Peinado VI, Go T, Schreiber C, von
Richthofen M, Bader A, Haag J, Darsow KH, Bartel SJ, Lange HA, Furlani D, Steinhoff G, Macchiarini P: Mesenchymal stem cells restore lung function by recruiting resident and nonresident proteins. Cell Transplant 2011; 20:1561-1574.

4 Central Ethical Review Board, expert group on misconduct in research. Statement Ref. No. O 16-2016 02/06/2017 (accessed April 4, 2018).

5 Karolinska Institutet, Dnr: 2-1764/2016. (The document can be requested at registrator@ ki.se.) 